\title{
Efficient Near-Infrared Photosensitizer with Aggregation-Induced Emission for Imaging-Guided Photodynamic Therapy in Multiple Xenograft Tumor Models
}

Jun Dai, ${ }^{\dagger, a}$ Yinghao Li, ${ }^{\dagger, b}$ Zi Long, ${ }^{c}$ Ruming Jiang, ${ }^{b}$ Zeyan Zhuang, ${ }^{b}$ Zhiming Wang, ${ }^{b}$ Zujin Zhao, ${ }^{* b}$ Xiaoding Lou, ${ }^{*}$ Fan Xia, ${ }^{c}$ and Ben Zhong Tang ${ }^{* b d}$

a Department of Obstetrics and Gynecology, Tongji Hospital, Tongji Medical College, Huazhong University of Science and Technology, Wuhan 430074, China.

b State Key Laboratory of Luminescent Materials and Devices, Guangdong Provincial Key Laboratory of Luminescence from Molecular Aggregates, South China University of Technology, Guangzhou 510640, China. E-mail: mszjzhao@scut.edu.cn

${ }^{c}$ Engineering Research Center of Nano-Geomaterials of Ministry of Education, Faculty of Materials Science and Chemistry, China University of Geosciences, Wuhan 430074, China. E-mail: louxiaoding@cug.edu.cn

d Department of Chemistry, The Hong Kong University of Science and Technology, Clear Water Bay, Kowloon, Hong Kong, China. E-mail: tangbenz@ust.hk

$\dagger$ These authors contribute equally to this work. 


\section{Contents}

Additional figures. 


\section{Additional figures}

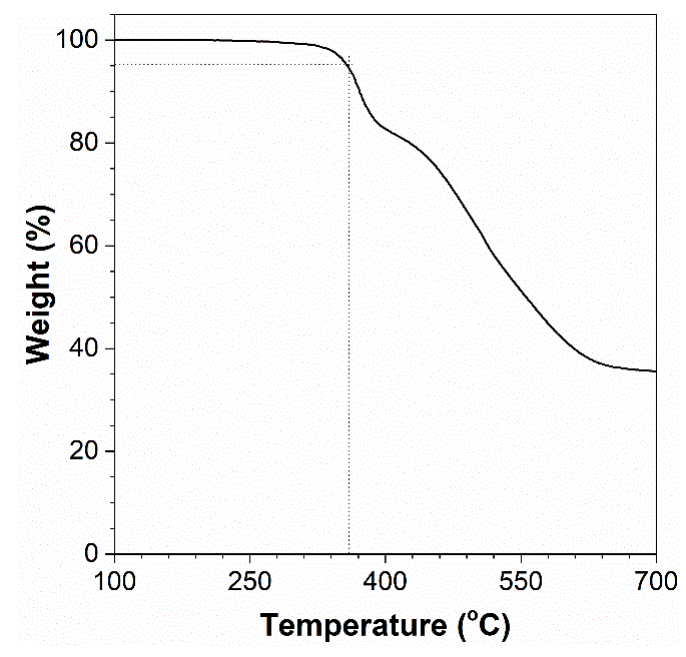

Figure S1. TGA curve of TTB, recorded under nitrogen at a heating rate of $10^{\circ} \mathrm{C} \mathrm{min}^{-1}$. TTB shows a high thermal decomposition temperature $\left(T_{\mathrm{d}}\right)$ at $357{ }^{\circ} \mathrm{C}$, corresponding to $5 \%$ loss of the initial weight, indicating its good thermal stability.

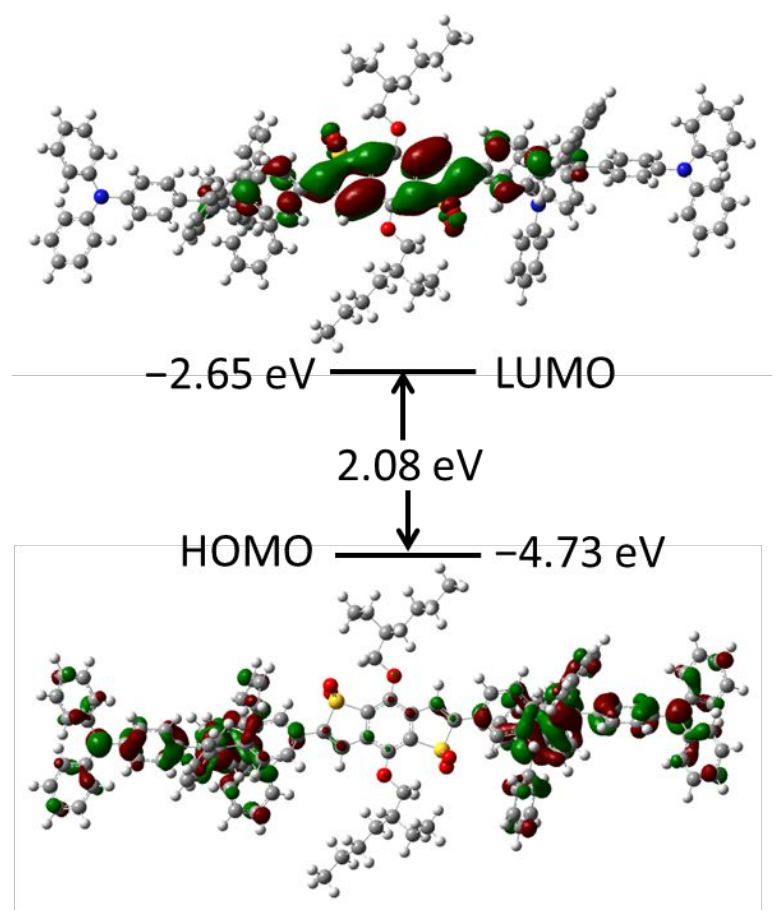

Figure S2. B3LYP/6-31G(d) calculated molecular orbital amplitude plots of HOMO and LUMO of TTB. 

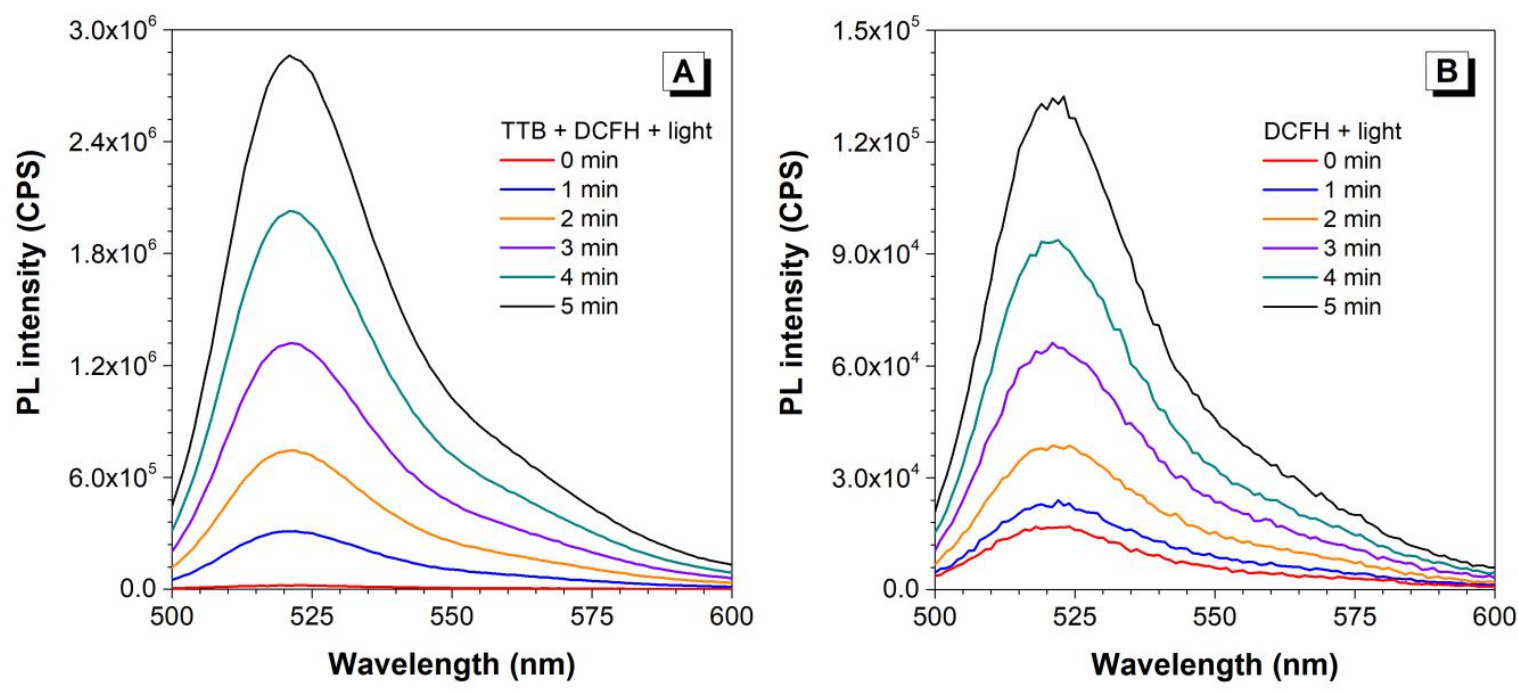

Figure S3. Fluorescence spectra for ROS with (A) or without (B) white light irradiation using DCFH as fluorescence probe. TTB concentration $\left(5 \times 10^{-6} \mathrm{M}\right.$ in PBS $)$, DCFH concentration $\left(1 \times 10^{-6} \mathrm{M}\right.$ in PBS), white light irradiation (400-700 nm, $\left.100 \mathrm{~mW} \mathrm{~cm}^{-2}\right)$.
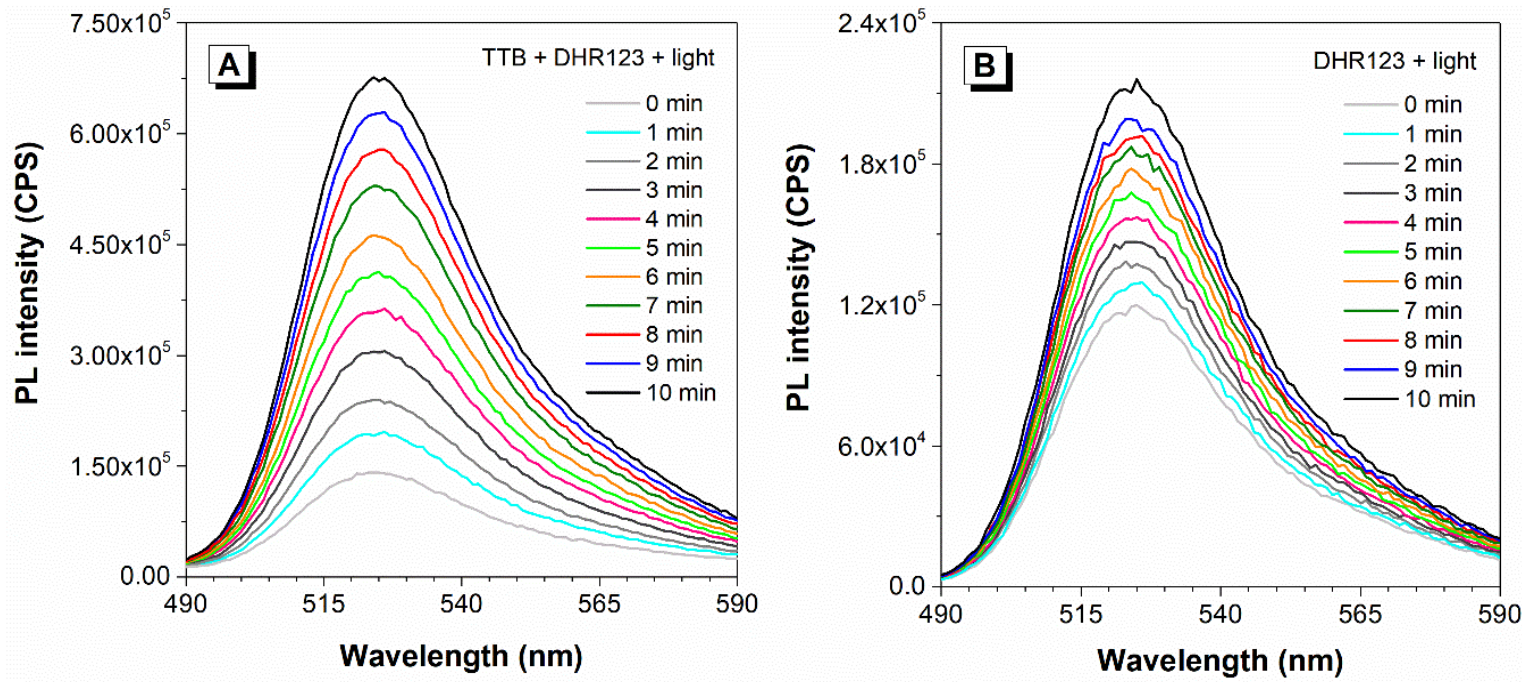

Figure S4. Fluorescence spectra for $\mathrm{O}_{2}^{\cdot-}$ with (A) or without (B) white light irradiation using DHR123 as fluorescence probe. TTB concentration $\left(5 \times 10^{-6} \mathrm{M}\right.$ in PBS), DHR123 concentration (2 $\times 10^{-6} \mathrm{M}$ in PBS), white light irradiation $\left(400-700 \mathrm{~nm}, 100 \mathrm{~mW} \mathrm{~cm}^{-2}\right)$. 


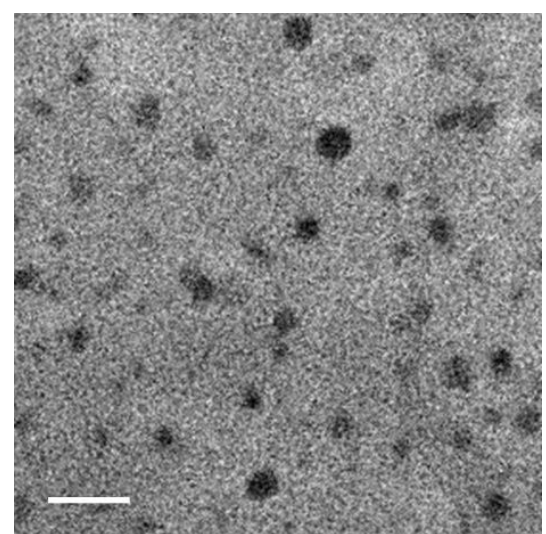

Figure S5. TEM image of RGD-4R-MPD/TTB NP. Scale bar: $0.2 \mu \mathrm{m}$.
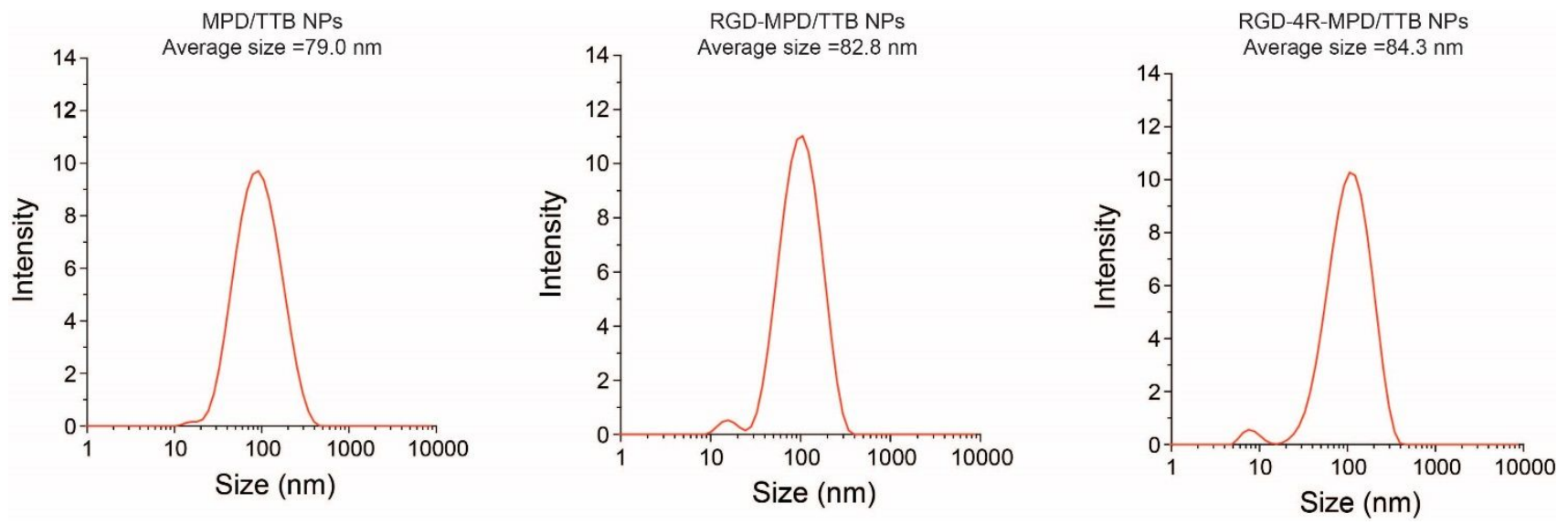

Figure S6. Hydrodynamic size distributions of MPD/TTB NPs, RGD-MPD/TTB NPs and RGD-4R-MPD/TTB NPs in aqueous solution containing 20\% serum. 


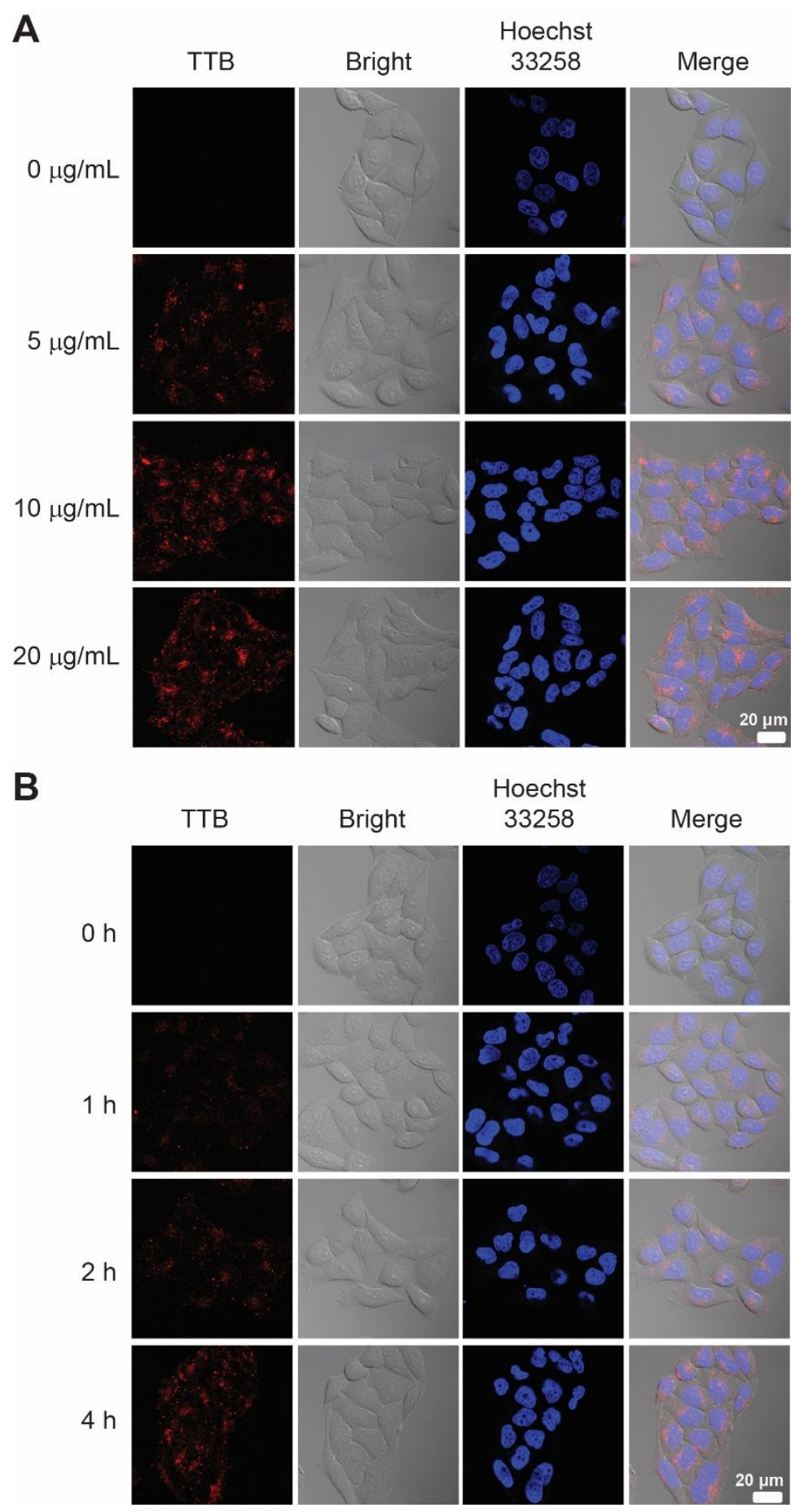

Figure S7. (A) PC3 cells were incubated with various concentrations of RGD-4R-MPD/TTB NPs for $4 \mathrm{~h}$. (B) PC3 cells were incubated with $10 \mu \mathrm{g} \mathrm{mL}-1$ RGD-4R-MPD/TTB NPs for various time $\left(\lambda_{\text {ex }}\right.$ $\left.=543 \mathrm{~nm}, \lambda_{\mathrm{em}}=660-720 \mathrm{~nm}\right)$. The nuclei were stained by Hoechst $33258\left(\lambda_{\mathrm{ex}}=720 \mathrm{~nm}, \lambda_{\mathrm{em}}=\right.$ $420-480 \mathrm{~nm})$. Scale bar: $20 \mu \mathrm{m}$. 
A

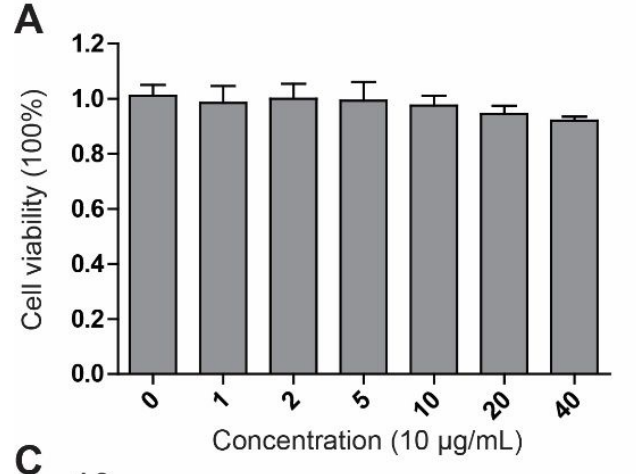

C

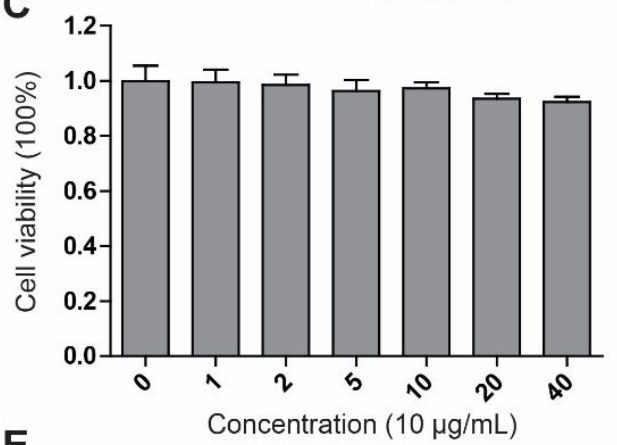

E
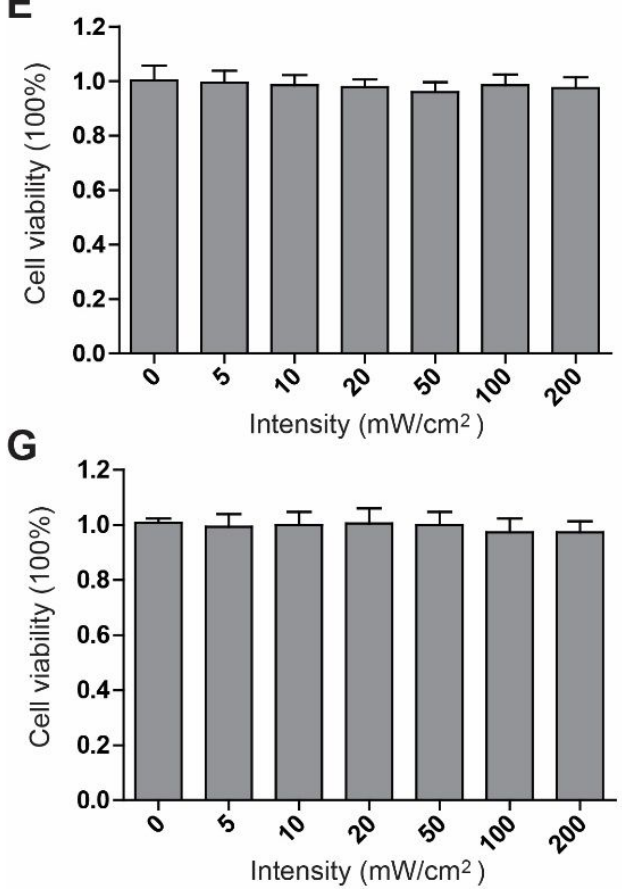

B

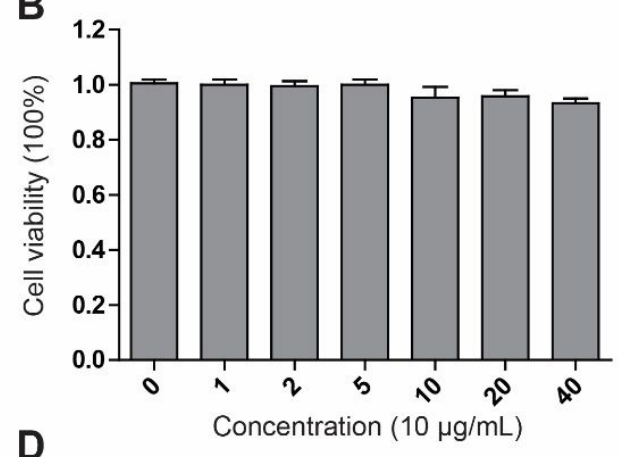

D

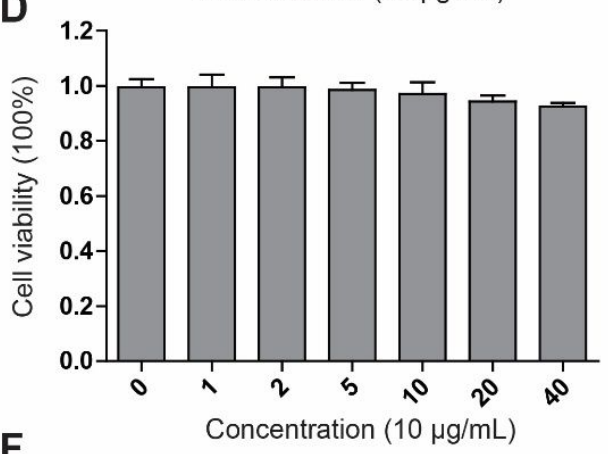

$\mathbf{F}$

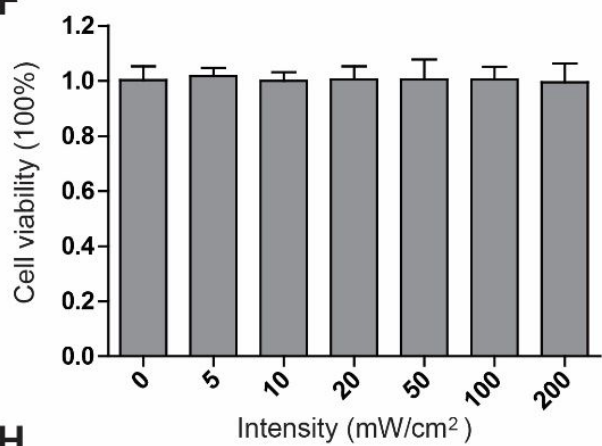

H

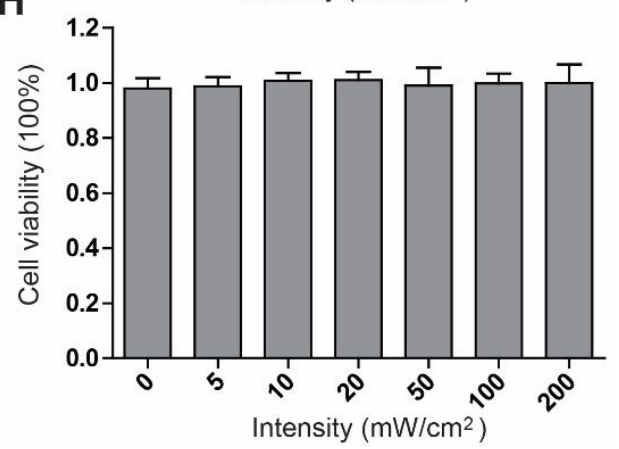

Figure S8. The CCK-8 assay was used to detect the cytotoxicity effect of different concentrations of RGD-4R-MPD/TTB NPs on (A) MCF7, (B) HeLa, (C) PC3 and (D) SKOV-3 cells. The CCK-8 assay was performed to test the cytotoxicity effect of different intensity of white light on (E) MCF7, (F) HeLa, (G) PC3 and (H) SKOV-3 cells. The data were presented as mean $\pm \mathrm{SD}(\mathrm{n}=3)$. 


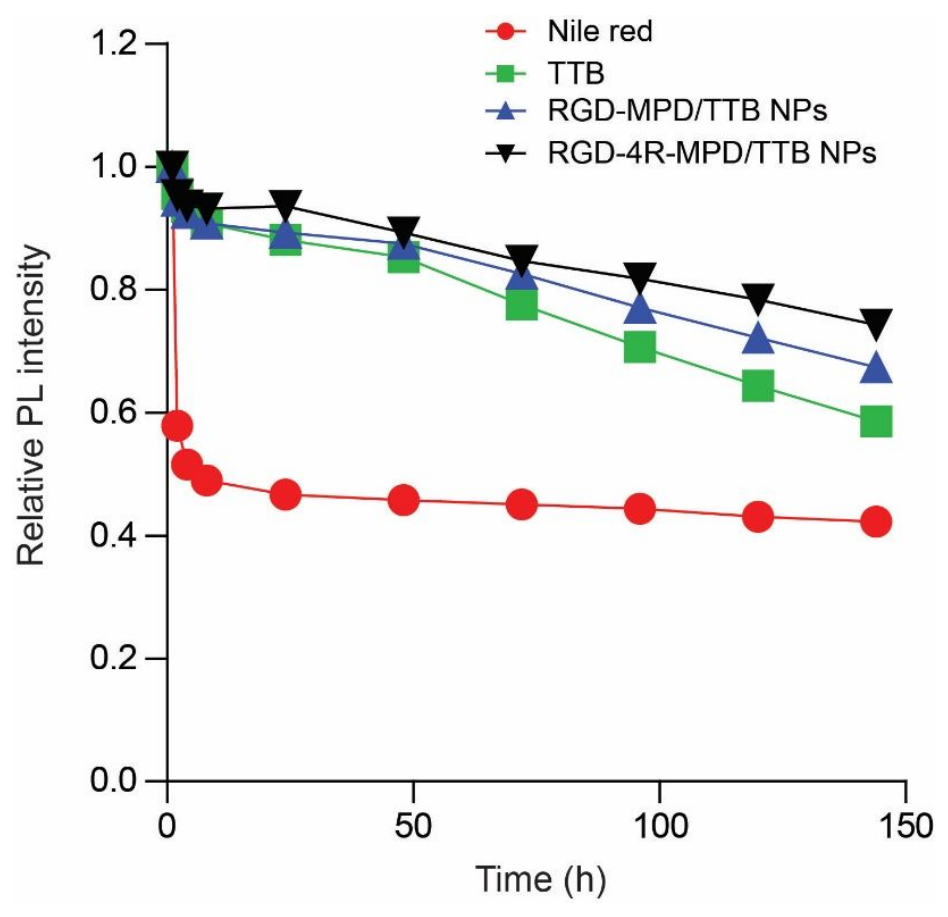

Figure S9. Relative fluorescence intensity of the tumors in PC3 tumor-bearing mice after intratumoral administration of Nile red, TTB, RGD-MPD/TTB NPs, and RGD-4R-MPD/TTB NPs, respectively. 


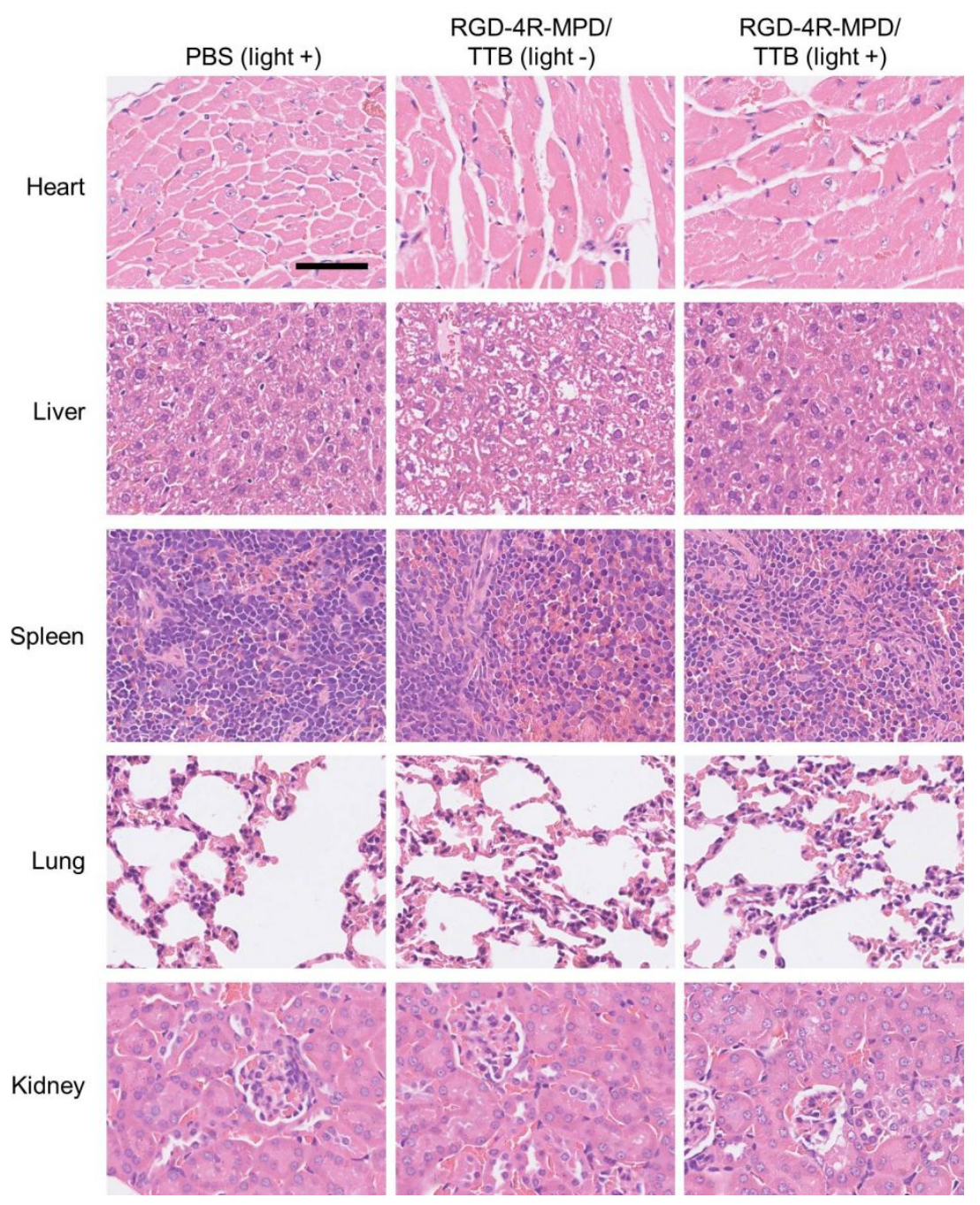

Figure S10. H\&E staining of heart, liver, spleen, lung and kidney in HeLa tumor-bearing mice are carried out after photodynamic therapy to assess the biocompatibility of RGD-4R-MPD/TTB NPs. Scale bar: $50 \mu \mathrm{m}$. 


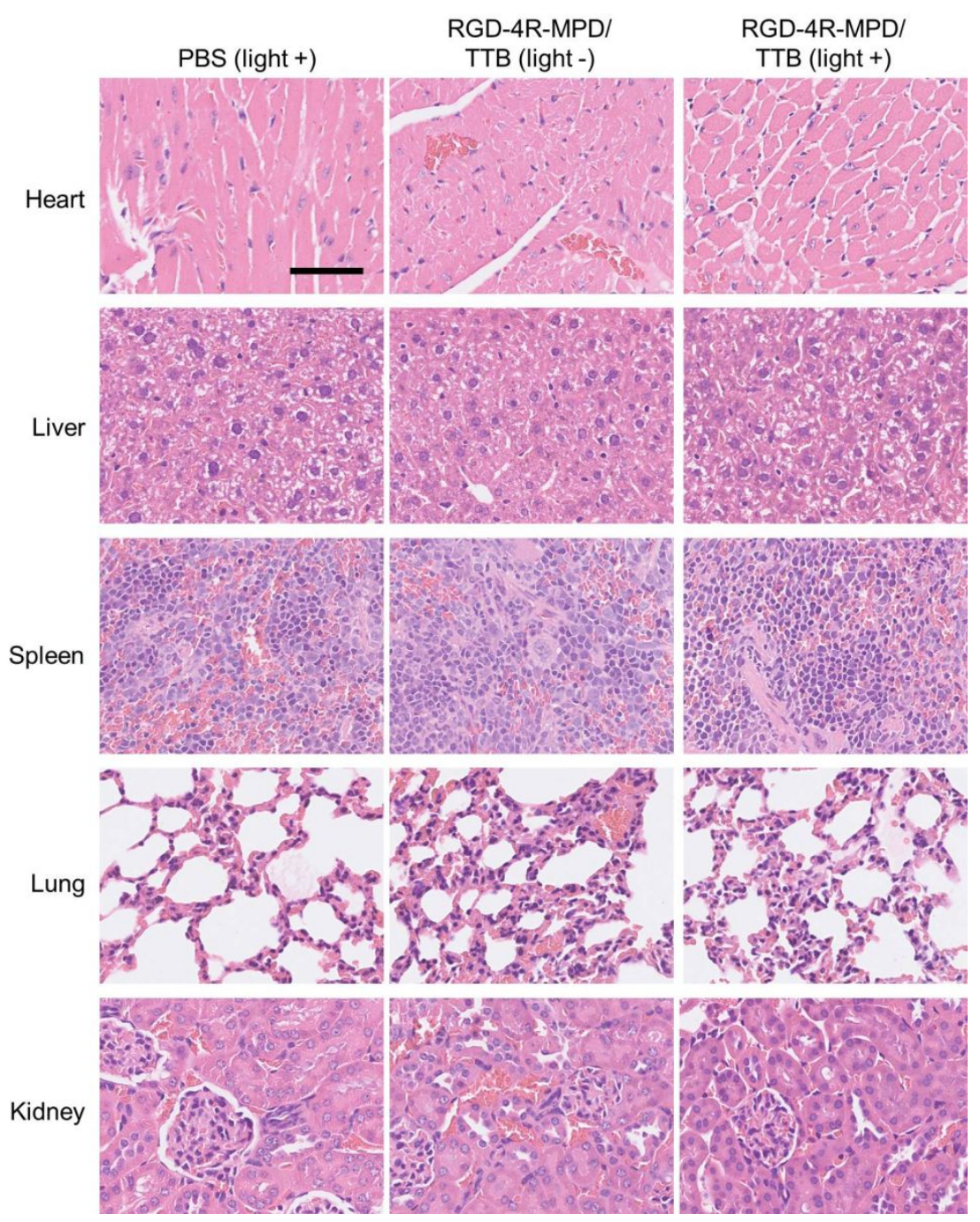

Figure S11. H\&E staining of heart, liver, spleen, lung and kidney in PC3 tumor-bearing mice are carried out after photodynamic therapy to assess the biocompatibility of RGD-4R-MPD/TTB NPs.

Scale bar: $50 \mu \mathrm{m}$. 


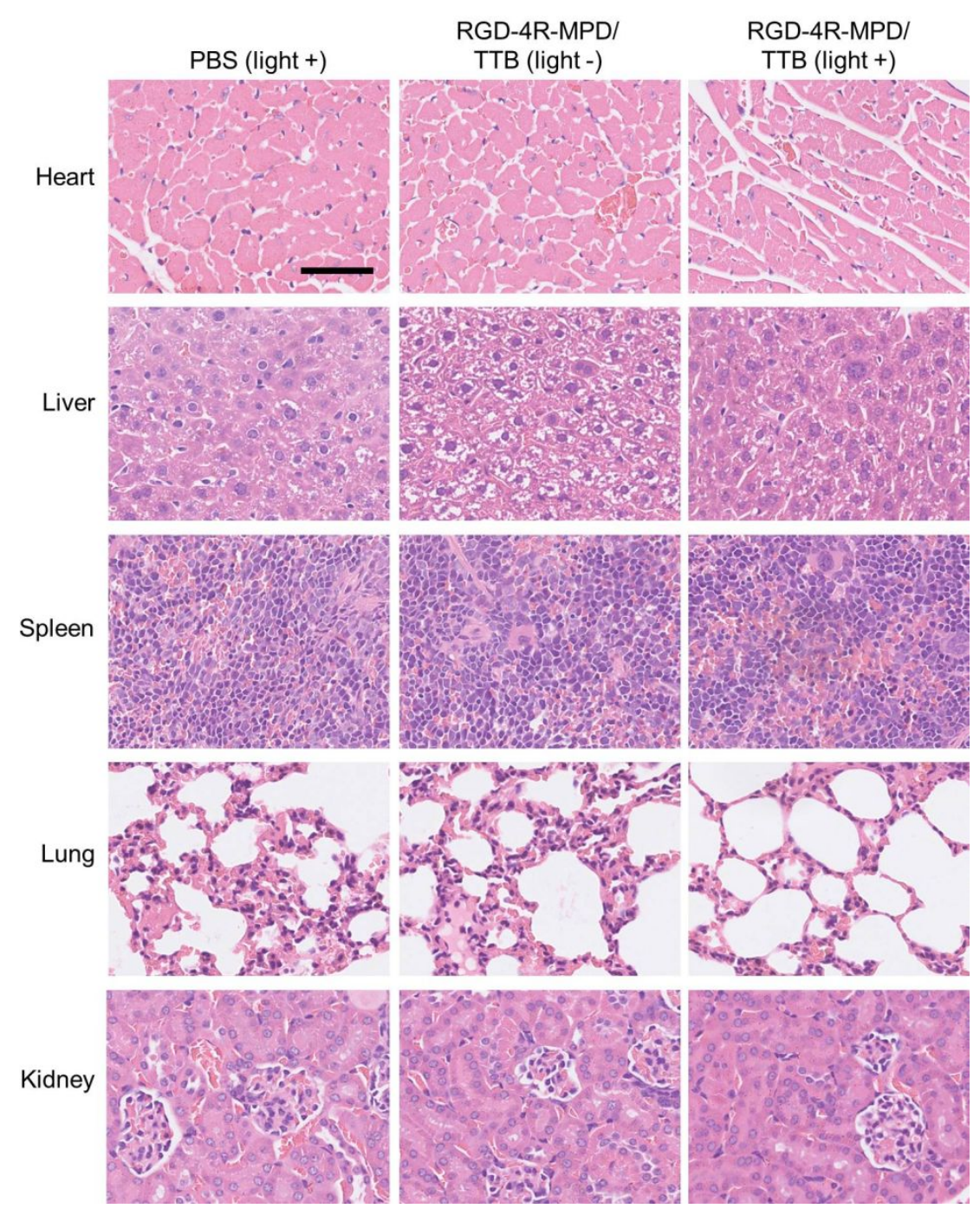

Figure S12. H\&E staining of heart, liver, spleen, lung and kidney in SKOV-3 tumor-bearing mice are carried out after photodynamic therapy to assess the biocompatibility of RGD-4R-MPD/TTB NPs. Scale bar: $50 \mu \mathrm{m}$. 